\title{
Multi-color imaging of in vivo with fluorescent proteins: the new cell biology.
}

\author{
Robert M. Hoffman
}

AntiCancer, Inc., 7917 Ostrow Street, San Diego, CA 92111

Imageable in vivo cell biology is being developed with multi-colored fluorescent proteins. With the use of fluorescent proteins, the behavior of cells can be visualized in the living animal. An example of the new cell biology is dual-color fluorescence imaging using red fluorescent protein (RFP)-expressing tumors transplanted in green fluorescent protein (GFP)-expressing transgenic mice $[1,2]$. These models show with great clarity the details of the tumor-stroma interaction especially tumor induced angiogenesis, tumorinfiltrating lymphocytes, stromal fibroblasts and macrophages. Another example is the color-coding of cells with RFP or GFP such that both cell types can be simultaneously visualized in vivo.

We have recently shown that the neural-stem-cell-associated protein nestin is expressed in hair-follicle bulge-area stem cells in transgenic mice with nestin-regulatory-elementdriven green fluorescent protein (ND-GFP). The ND-GFP stem cells were shown to give rise to the outer-root sheath of the hair follicle as well as to a nestin-expressing interfollicular vascular network. In the present study, we demonstrate that keratin 15negative ND-GFP-expressing stem cells in the hair follicle bulge area can differentiate into neurons, glia, keratinocytes, smooth muscle cells, and melanocytes in vitro. These pluripotent nestin-GFP-expressing stem cells are CD34-positive, as well as keratin 15negative, suggesting their relatively undifferentiated state. The apparent primitive state of the ND-GFP stem cells enable their pluripotency. Furthermore, we show that NDGFP-expressing stem cell-derived cells differentiate into neurons after transplantation to the subcutis of nude mice. These results suggest that hair follicle bulge-area ND-GFP stem cells may provide an accessible, autologous source of undifferentiated multipotent stem cells for therapeutic application $[3,4]$.

Dual-color cells expressing GFP in the nucleus and RFP in the cytoplasm enable realtime visualization of nuclear-cytoplasm dynamics including cell cycle events and apoptosis. Dual-color cells also enable the in vivo imaging of cell and nuclear deformation as well as trafficking in capillaries in living animals. Multiple-color labeling of cells will enable multiple events to be simultaneously visualized in vivo including gene expression, ion fluxes, protein and organelle trafficking, chromosome dynamics and numerous other processes currently still only studied in vitro $[5,6]$.

Using whole-body real-time fluorescence imaging to detect GFP-expressing human multiple myeloma (MM) cells in SCID-NOD mice, we have serially monitored the development and progression of MM in bone and other organs. Major involvement of the axial skeleton, including spine, skull, and pelvis) and frequent development of paralysis due to spinal lesions was observed by whole-body imaging (7). 
A PTHrP expressing PC-3-GFP human prostate cancer variant was used to determine the efficacy of bisphophonates on tumor growth in the skeleton of nude mice. The PC-3 cells produced extensive bone lesions when injected into the tibia. Bone growth of the PC-3 cells could be monitored by whole-body GFP imaging, X-ray, and by measurements of tumor products in serum, notably PTHrP. The bisphophonate, Pamidronate, reduced tumor burden as assessed by GFP imaging and biomarkers (8). We have now identified a novel effective bisphosphonate from a group of seven bisphosphonates in our current collection. The area of GFP fluorescence in the anterior tibia of the bisphosphonatetreated and untreated control mice was compared two months after the injection of PC-3GFP human prostate cancer cells. The size of the GFP lesions significantly decreased in the positive-control Pamidronate-treated mice and novel-bisphosphonate-treated mice compared to the controls ( $\mathrm{p}<0.05$ and 0.01 , respectively).

\section{References}

1. $\quad$ Yang, M., et al., Cancer Research 64 (2004) 8651-8656.

2. $\quad$ Yang, M., et al., Proc. Natl. Acad. Sci. USA 100 (2003) 14259-14262.

3. $\quad$ Li, L., et al., Proc. Natl. Acad. Sci. USA 100 (2003) 9958-9961.

4. $\quad$ Amoh, Y., et al., Proc. Natl. Acad. Sci. USA 101 (2004) 13291-13295.

5. $\quad$ Yamamoto, N., et al., Cancer Research 63 (2003) 7785-7790.

6. Yamamoto, N., et al., Cancer Research 64 (2004) 4251-4256.

7. $\quad$ Mitsiades, C.S., et al., Cancer Research 63 (2003) 6689-6696.

8. $\quad$ Burton, et al., The Prostate 62 (2005) 275-281.
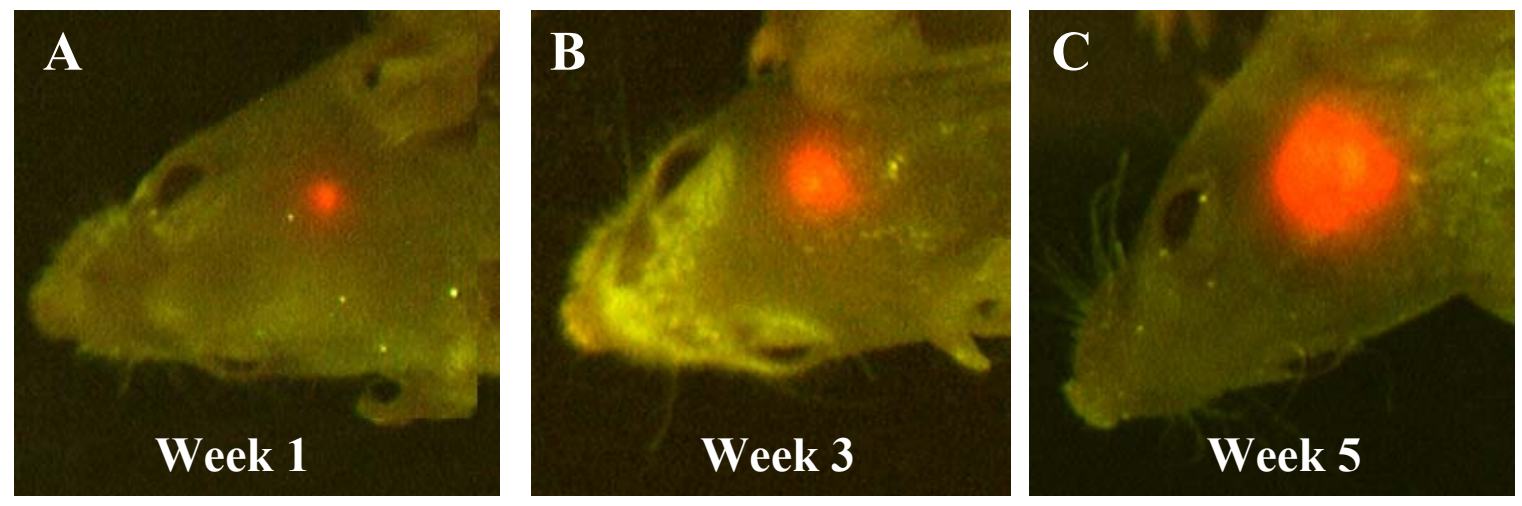

Figure 1. Real time, whole-body imaging of U87-RFP human glioma growing in the brain of a nude mouse. The RFP-expressing human glioma was transplanted by surgical orthotopic implantation (SOI) in the right parietal lobe of the nude mouse brain. The images were acquired externally and non-invasively. A: U87-RFP tumor growing in the brain one week after SOI. B \& C: U87-RFP tumor growing in the brain 3 and 5 weeks after SOI, respectively. 